\title{
Results and Perspectives in Forward Physics with ATLAS
}

\author{
B. Giacobbe on behalf of the ATLAS Collaboration \\ Istituto Nazionale di Fisica Nucleare, Sezione di Bologna, Italy
}

\begin{abstract}
A review of the ATLAS forward physics results is given with particular emphasis on the aspects of relevance for the cosmic rays community. These include proton-proton cross section measurements at $\sqrt{s}=7 \mathrm{TeV}$, diffractive physics studies using rapidity gaps, measurements of energy flow as a function of pseudorapidity, and the first cross section measurement performed in the recently started Run 2 at $\sqrt{s}=13 \mathrm{TeV}$. The ATLAS future perspectives will also be discussed, focused on the phase 1 upgrade project AFP, underlying its potential for a wide forward physics program both at low and high luminosity.
\end{abstract}

Keywords: Forward Physics, Cosmic Rays, Monte Carlo, Hadronic Cross Section, Diffraction

\section{Introduction}

One of the still open items in cosmic ray physics is the interpretation of the cosmic particle flux spectrum measured by the experiments. The observation of cosmic rays spreads over a very large energy interval (up to $10^{20} \mathrm{eV}$ ) and, in particular for the ultra-high energy range, it is performed by large area experiments at the earth-surface observing the showers produced by primary cosmic particles interacting with the atmosphere. At $E \sim 10^{16} \mathrm{eV}$ the spectrum shows a steepening, known as the knee, while at $E \sim 10^{19} \mathrm{eV}$ an additional feature appears, called the ankle (see Figure 1). The knee is usually associated with the maximum particle acceleration of galactic origin, while the ankle with extragalactic sources. The results from the various experiments in this energy region show important discrepancies both in terms of absolute flux and in terms of mass composition of the primary particles. The energy and mass composition of the primaries is determined from the energy and shape of the shower, as measured at the surface of the earth, through hadronic Monte Carlo (MC) simulations of the shower development in the atmosphere. The involved processes are dominated by forward, soft QCD interactions, poorly known at these energies. From Figure 1 one can see that the proton- proton interactions at the LHC at $\sqrt{s}=7$ and $13 \mathrm{TeV}$, correspond to the energy range of cosmic rays at the knee. Detailed measurements of the proton-proton interaction in the forward region are therefore a valuable input for the cosmic rays community as they can be used to tune the parameters of the models used for the description of the cosmic showers. In this context, ATLAS has performed a wide set of measurements, including the proton-proton cross sections (both total, elastic and inelastic), the energy flow distribution as a function of the particles pseudorapidity, and jet production. Such a program can be perfomed thanks to a large set of forward detectors (described in section 2) aimed to detect particles emerging from the pp interaction close or even inside the beam-pipe at large pseudorapidity. In sections 3 and 4 the pp cross section measurements are described using different techniques, while in section 5 the energy flow measurement is discussed. In section 6 the future perspectives of forward physics in ATLAS are discussed including the new AFP detector, which represents one of the major ATLAS upgrades for the LHC Run 2. 


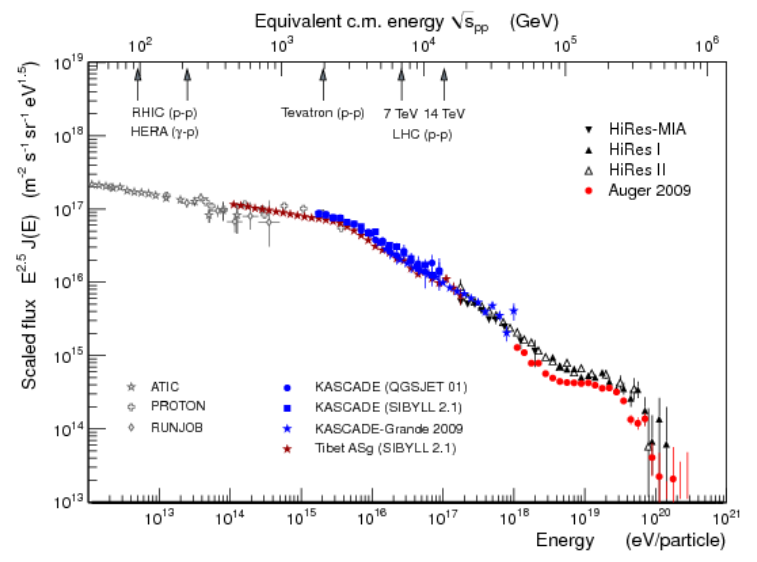

Figure 1: Energy distribution of cosmic rays above $10^{12} \mathrm{eV}$. At $E \sim$ $10^{16} \mathrm{eV}$ and $E \sim 10^{19} \mathrm{eV}$ respectively, the knee and the ankle are visible. The equivalent pp-collider center of mass energy is shown in the upper horizontal axis. The LHC center of mass energy in Run 1 is explicitly reported. In Run 2, LHC decided at the end to run at a center of mass energy of $13 \mathrm{TeV}$ instead of $14 \mathrm{TeV}$. Taken from Ref. [1].

\section{The ATLAS Forward Detectors}

ATLAS (Ref. [2]) is a multi-purpose detector operating at the Large Hadron Collider (LHC), designed to study proton-proton interactions at the TeV scale. ATLAS can perform a wide set of forward physics measurements thanks to the various dedicated subdetectors, covering the range of large pseudorapidity. LUCID and $\mathrm{BCM}$ are dedicated to the luminosity determination, and are not discussed in this paper (all details can be found in Ref. [3]), as well as the Zero Degree Calorimeter (ZDC) dedicated to heavy ions physics. ALFA (Absolute Luminosity for ATLAS, Ref. [4]) is designed to measure small-angle proton scattering at a pseudorapidity $|\eta|>8.2^{1}$. It is composed by two stations, located at 238 and 241 meters on both sides of the interaction point (IP). The detectors are housed into Roman Pots which can be moved inside the beam pipe, close to the proton beam, without breaking the LHC vacuum, in order to detect protons at scattering angles as low as $10 \mu \mathrm{rad}$. Elastically scattered protons are detected in the Main Detectors (MDs) made of scintillating fibers with a measured spatial resolution of about $30 \mu \mathrm{m}$ and an efficiency

\footnotetext{
${ }^{1}$ ATLAS uses a right-handed coordinate system with its origin at the nominal IP in the centre of the detector and the $\mathrm{z}$-axis along the beam pipe. The X-axis points from the IP to the centre of the LHC ring, and the $y$-axis points upwards. Cylindrical coordinates $(r, \phi)$ are used in the transverse plane, $\phi$ being the azimuthal angle around the $\mathrm{z}$-axis. The pseudorapidity is defined in terms of the polar angle $\theta$ as $\eta=-\ln \tan (\theta / 2)$.
}

to detect protons of $93 \%$ per fiber. Additional Overlap Detectors are attached to the MDs in order to measure their relative distance. The detectors are complemented by trigger counters. The Minimum Bias Trigger Scintillator detector (MBTS, Ref. [3]) is composed by high efficiency scintillation counters, placed at about 3.6 meters on both sides of the IP, and covers a pseudorapidity interval $2.1<|\eta|<3$.8. Each module is organised into 2 disks (inner and outer) each composed by 8 independent sectors. The light produced by each counter is guided into a photomultiplier tube by wavelength-shifting optical fibers. The detector shows an efficiency for inelastic events close to $100 \%$.

\section{Proton-Proton Cross Sections at $\sqrt{s}=7 \mathrm{TeV}$}

The total proton-proton cross section is a fundamental parameter of strong interactions, defining the size of the interaction region as a function of the energy. Quantum chromodinamics (QCD) cannot at present provide a precise calculation of the cross section, as large distance, non-perturbative interactions are involved in the collision process, but only estimations or bounds can be set based on high-energy scattering relations. Among these the optical theorem relates the imaginary part of the forward-elastic scattering amplitude to the total cross section, thus relating the elastic cross section at zero momentum transfer to the total cross section:

$$
\sigma_{\text {tot }}^{2}=\left.\frac{16 \pi(\hbar c)^{2}}{1+\rho^{2}} \frac{d \sigma_{\mathrm{el}}}{d t}\right|_{\mathrm{t} \rightarrow 0}
$$

In general the total cross section $\sigma_{\text {tot }}$ can be decomposed into an elastic $\left(\sigma_{\mathrm{el}}\right)$ and an inelastic $\left(\sigma_{\text {inel }}\right)$ parts. The inelastic component is further made of a non-diffractive component $\sigma_{\mathrm{ND}}$ (dominated by exchange of color degrees of freedom) and a diffractive one $\sigma_{\mathrm{D}}$ (where the particles exchanged by the interacting protons are colorless). Experimentally, the elastic events can be identified by observing the two interacting protons emerging intact and practically udeflected from the IP (protons tag). On the other hand, in diffractive events one proton (single-diffraction, SD) or both (double-diffraction, DD) dissociate into a mixture of low diffractive mass particles of mass $M_{\mathrm{X}}$. The typical experimental signature of single diffractive events is the presence of one intact proton in one side of the detector and some activity, due to the products of the dissociated proton, on the opposite side, with an empty region separating them (rapidity gap). Rapidity gaps also characterize double diffractive events where both ends of the detector detect the products of the two dissociated protons. 
ATLAS performed a wide set of measurements at $\sqrt{s}=7 \mathrm{TeV}$, using different and independent experimental techniques, in order to provide an as much as possible complete picture of the various cross section components. This is the only way to provide a good understanding of the soft processes which by far dominate the proton-proton interaction. Moreover the experimental input is a valuable tool to help tuning, with both hard and soft processes, the MC models used, among other, to simulate the development of the showers initiated by cosmic rays in the atmosphere.

\subsection{Total Inelastic Cross Section}

The measurement of the inelastic cross section was first measured by ATLAS in a low luminosity run with a total integrated luminosity of $20.3 \pm 0.7 \mu \mathrm{b}$, in the kinematic range $\xi=M_{\mathrm{X}}^{2} / s>5 \cdot 10^{-6}\left(M_{\mathrm{X}}>15.7 \mathrm{GeV}\right)$ and then extrapolated to the full phase space (Ref. [5]). The fiducial region is defined by the geometrical acceptance of the MBTS used to trigger and select offline the events by requiring at least two counters to be hit. A further requirement that the two hit counters belong to the same side of the IP (single-sided events selection) is used to constrain, through a comparison with the MC generators used to simulate the events, the diffractive component. The inelastic cross section is calculated as:

$$
\sigma_{\text {inel }}\left(\xi>5 \cdot 10^{-6}\right)=\frac{N-N_{\mathrm{bg}}}{\epsilon_{\text {trig }} \cdot L} \cdot \frac{1-f_{\xi<5 \cdot 10^{-6}}}{\epsilon_{\text {sel }}}
$$

where $N$ and $N_{\text {bg }}$ are the number of selected and background events, $f_{\xi<5 \cdot 10^{-6}}$ is the number of events passing the selection although out of the acceptance, $\mathrm{L}$ is the total integrated luminosity and $\epsilon_{\text {trig }}$ and $\epsilon_{\text {sel }}$ are the trigger and selection efficiencies. $N_{\text {bg }}$ and $\epsilon_{\text {trig }}$ are directly obtained from data and used to tune the MC simulation. Various MC generators are used to describe the properties of the inelastic collisions, namely Pythia6 (Ref. [6]), Pythia8 (Ref. [7]) and Phojet (Ref. [8]), and the generated events are then passed through the official ATLAS simulation based on Geant4. All generators distinguish among ND, SD and DD processes but they provide different predictions of the various cross section fractions and dependence on the $\xi$-acceptance. The tuned MCs are used to evaluate $\epsilon_{\text {sel }}$ and $f_{\xi<5 \cdot 10^{-6}}$. The generators are also tuned measuring the fraction of single-sided events $R_{\mathrm{ss}}$ with respect to the entire sample: according to all MC generators, single-sided events are of diffractive origin in $99 \%$ of all cases. The measured value $R_{\mathrm{ss}} \sim 10 \%$ is used to constrain the diffractive fraction $f_{\mathrm{D}}=\sigma_{\mathrm{D}} / \sigma_{\text {inel }}$ given by the various $\mathrm{MC}$ models, and provides, for the default Pythia8, a value $f_{\mathrm{D}}=26.9_{-1.0}^{+2.5} \%$ (see Figure 2). In Figure 3 the results of the measurement for the fiducial and total (extrapolated) inelastic cross sections are shown, compared to various theoretical models. The results are:

$$
\begin{aligned}
& \sigma_{\text {inel }}\left(\xi>5 \cdot 10^{-6}\right)=60.22 \pm 2.1 \mathrm{mb} \\
& \sigma_{\text {inel }}(f u l l)=69.1 \pm 2.4 \pm 6.9_{\text {extr }} \mathrm{mb}
\end{aligned}
$$

The fiducial value is significantly lower than both Pythia and Phojet predictions, while the total cross section agrees with the Pythia predictions. The large discrepancy among the various MC generators for what concerns the extrapolation to the full acceptance entirely determines the extrapolation uncertainty. This problem is avoided in the measurement of the inelastic cross section discussed in section 3.3.

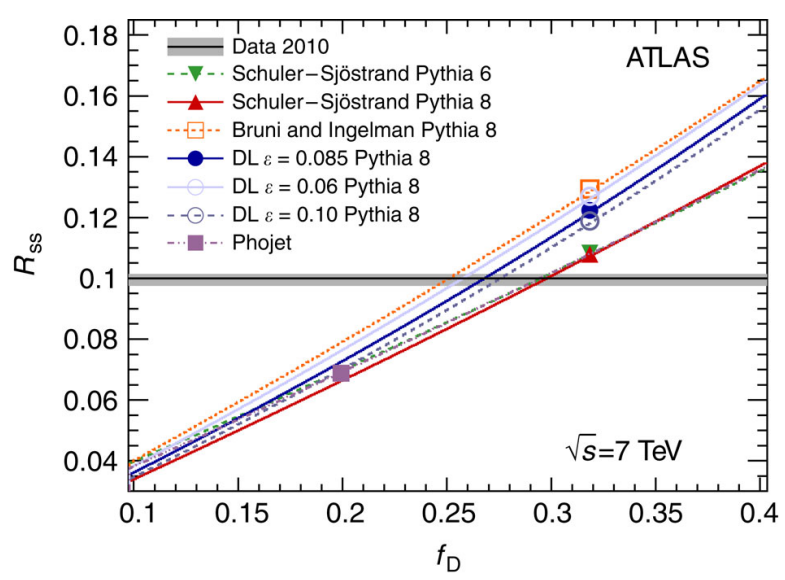

Figure 2: Dependence of the fraction of single-sided events $R_{\mathrm{Ss}}$ on the relative diffractive contribution $f_{\mathrm{D}}$. Taken from Ref. [5].

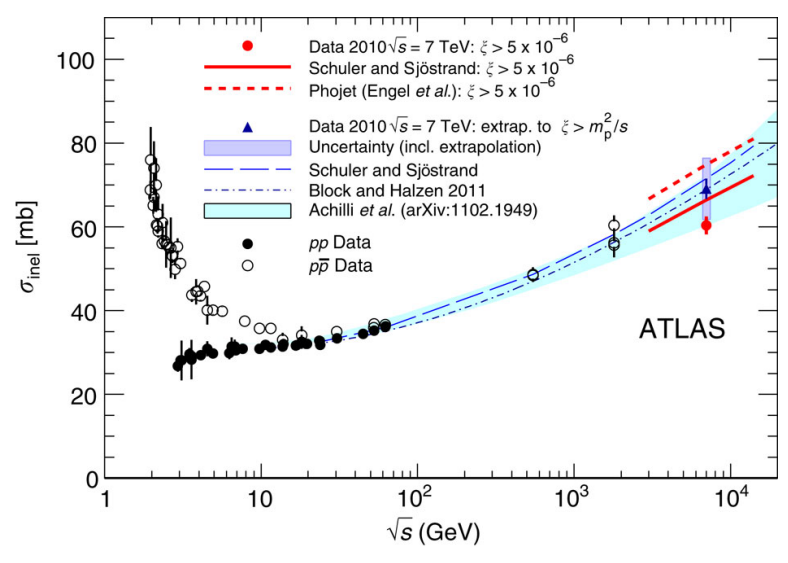

Figure 3: The inelastic cross section versus $\sqrt{s}$. Taken from Ref. [5]. 


\subsection{Differential Inelastic Cross Section}

Diffractive cross sections at high energy are one of the most relevant quantities of interest for the cosmic rays community. The study of such low-multiplicity events have a strong impact on the understanding of airshowers induced by cosmic particles. In the previous section the measurement of $\sigma_{\text {inel }}$ and its diffractive fraction $f_{\mathrm{D}}$ was presented. In this section a measurement of the differential features of the inelastic (and therefore also diffractive) cross section is presented and the comparison with the predictions of the most generally used MC generators is discussed (Ref. [9]). The cross section is measured in terms of $\Delta \eta^{F}$, the larger of the pseudorapidity regions extending to the forward limits of the ATLAS sensitivity $(\eta= \pm 4.9)$ in which no final state particles above a certain $p_{\mathrm{T}}$ are produced (the $p_{\mathrm{T}}$ threshold is varied from 200 to $800 \mathrm{MeV}$ ). The measurements span over $0<\Delta \eta^{F}<8$, corresponding, for diffractive events, to $10^{-6}<\xi=M_{\mathrm{X}}^{2} / s<10^{-2}$ or $7<M_{\mathrm{X}}<700 \mathrm{GeV}, M_{\mathrm{X}}$ being the mass of the diffracted system.

All MC generators predict that non-diffractive processes populate the $\Delta \eta^{F}=0$ bin, with larger gaps occurring due to rapidity fluctuations in final state particle production, leading to an exponential suppression with rapidity gap size. Diffractive processes are on the contrary expected to populate the large $\Delta \eta^{F}$ region and, in particular, all generators predict the differential inelastic cross section to show a plateau for $\Delta \eta^{F}>\sim 3$. The Pythia and Phojet generators were used for correcting the data for experimental effects and for comparison with the corrected data. All of them implement different models for the diffractive dissociation processes.

The data were acquired at very low luminosity in order to minimize multiple collisions per bunch crossing, for a total integrated luminosity of $7.1 \pm 0.2 \mu \mathrm{b}^{-1}$, requiring at least one hit in the MBTS trigger system. Rapidity gaps are defined based on the combined information of the inner detector and, beyond its acceptance $(2.5<\eta<4.9)$, of the calorimeters.

In Figure 4 a comparison between the measured differential cross section is shown with the Pythia8 generator predictions (including the non-diffractive and the various diffractive components). An overall overestimation by the $\mathrm{MC}$ of $\sigma_{\text {inel }}$ can be observed. The generator generally describe the overall trend, but not the details, of the measured distribution. In particular the plateau at large rapidity gaps is indeed observed, but the raise at very large $\Delta \eta^{F}$ cannot be reproduced by the MC. The same observations hold for the Phojet generator (not shown). In order to reproduce the behaviour of the cross section for $\Delta \eta^{F}>\sim 5$, a modified model, implementing the Donnachie-Landshoff Pomeron flux with intercept $\alpha_{\mid \mathrm{P}}=1.058$, is used, with the result of improving the description at very large $\Delta \eta^{F}$, at the price of loosing predictivity in the intermediate region, probably due a non perfect description of the hadronization fluctuation of non-diffractive events (see Figure 5).

In Figure 6, the integral of the cross section from zero to a maximum gap-size is shown, as a function of the maximum gap size, and is compared to the measurement discussed in the previous section. A good consistency among the two measurements can be observed. In the figure the direct measurement in the full phase space performed by TOTEM (Ref. [10]) is also shown. Using this result, the integrated inelastic cross section for $\xi<8 \cdot 10^{-6}$ turns out to be $\sim 14.5 \mathrm{mb}(\sim 11 \mathrm{mb}$ if compared to the ATLAS value discussed in the next section), significantly larger than predicted by Pythia $(\sim 6 \mathrm{mb})$ and Phojet $(\sim 3 \mathrm{mb})$.

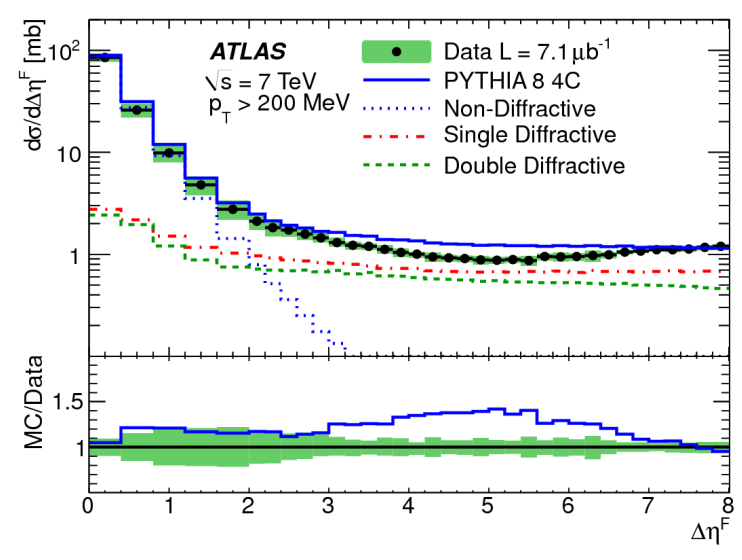

Figure 4: Inelastic cross section differential in forward gap size $\Delta \eta^{F}$ compared to the predictions of the Pythia8 generator. Taken from Ref. [9].

\subsection{Cross Sections with Proton Tag}

The measurements of the inelastic cross section presented in sections 3.1 and 3.2 suffer from large extrapolation uncertainties. Only a comprehensive approach including the measurement of the total and elastic cross section can avoid this limit. ATLAS has performed a measurement of the total pp cross section (Ref. [4]) by measuring the elastic scattering cross section in the forward direction within the Optical Theorem approach (Eq. 1). From the total and elastic cross section one can get a precise evaluation of the inelastic one.

According to Eq. 1, the experimental procedure is to measure the elastic scattering event rate, differential in 


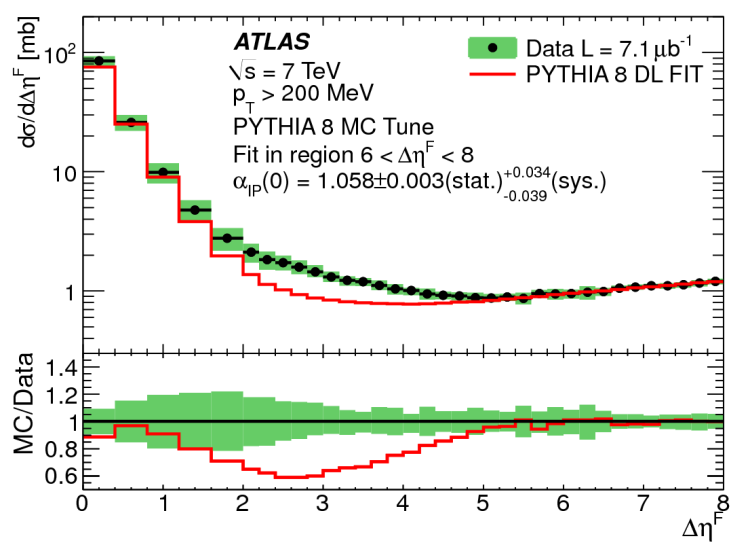

Figure 5: Inelastic cross section differential in forward gap size $\Delta \eta^{F}$ compared to the predictions of the Pythia8 generator including the modified Donnachie-Landshoff Pomeron flux. Taken from Ref. [9].

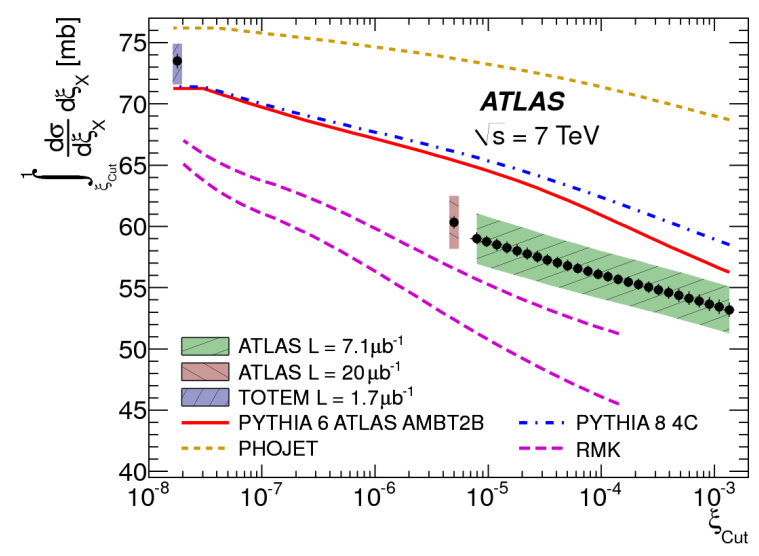

Figure 6: Inelastic cross section integrated from gap size of zero to a maximum variable. Taken from Ref. [9].

$|t|$ and extrapolate to $|t|=0$, by detecting the two practically undeflected protons emerging in the very forward direction. This requires a detector able to detect protons at scattering angles as low as $10 \mu \mathrm{rad}$. This is done by the ALFA detector, thanks to its large distance from the IP and to its capability to approach the beam to about 5 $\mathrm{mm}$. Moreover the data taking requires special LHC optics conditions, namely: 1) a high $\beta^{*}$ function at the IP (90 meters for this data taking) implying a weak beam focusing, i.e. small beam divergence, and 2) a parallelto-point focusing which allows to directly infer the protons scattering angle from the hit-position in the detector, independently of the production vertex position. This is achieved in the vertical plane only ( $y$-direction) and simplifies, at least in this plane, the reconstruction of the scattering angle from the measured position in the tracking system, while in the other direction $(x)$ the full beam-transport matrix has to be used for converting the hit-positions into scattering angles. From the scattering angles $\theta_{\mathrm{x}}$ and $\theta_{\mathrm{y}}$ the four-momentun trasfer $t$ is defined to be:

$$
-t=(\theta \cdot p)^{2}
$$

where $p$ is the nominal beam momentum of LHC, $3.5 \mathrm{TeV}$, and the differential elastic scattering distribution is measured.

In order to select pure elastic scattering events, a trigger is used requiring a coincidence between either of the two upper (lower) detectors on one of the two sides of the interaction point, and either of the two lower (upper) detectors on the opposite side. This back-to-back topology is the golden signature of the elastic events, while the anti-elastic topology is used for the background subtraction with data. Also the efficiency evaluation is carried out using data, while the transport matrix is obtained using MADx beam optics calculation package, and the unfolding procedure is done using the Pythia8 elastic generator.

In Figure 7 the measured elastic cross section differential in $-t$ is shown with a fit of the theoretical prediction with $\sigma_{\text {tot }}$ (the total pp cross section) and $B$ (the nuclear slope parameter) as free parameters. In the lower box the deviation of the points to the fit is shown. The fit is performed in the range from $-0.01<t<-0.1 \mathrm{GeV}^{2}$, where the lower values is chosen as low as possible to minimize extrapolation uncertainties still having a seizable efficiency, while the upper limit is chosen such to exclude the region where deviations from the single exponential function are expected. By integrating the differential elastic cross section also the total elastic cross section is measured, and by difference, the inelastic one can be inferred:

$$
\begin{aligned}
& \sigma_{\text {tot }}=95.35 \pm 0.38_{\text {stat }} \pm 1.25_{\text {sys }} \pm 0.37_{\text {extr }} \mathrm{mb} \\
& \sigma_{\text {el }}=24.00 \pm 0.19_{\text {stat }} \pm 0.57_{\text {sys }} \mathrm{mb} \\
& \sigma_{\text {inel }}=71.34 \pm 0.36_{\text {stat }} \pm 0.83_{\text {sys }} \mathrm{mb}
\end{aligned}
$$

In Figure 8 the total and elastic cross sections are compared to other published measurements and model predictions as a function of the center-of-mass energy. The measurement of $\sigma_{\text {inel }}$ is significantly more precise than the previous ATLAS measurements and the most precise among the LHC measurements at this energy. The comparison with TOTEM results (Ref. [11]) shows an agreement at the 1.3 (1.1) $\sigma$ level for the total (elastic) cross sections respectively. 


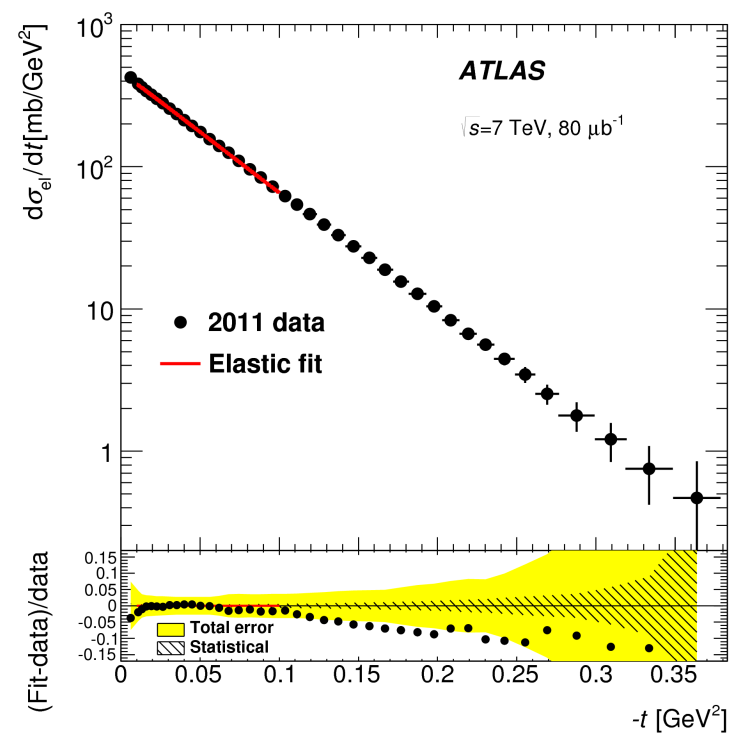

Figure 7: A fit of the theoretical prediction, with $\sigma_{\text {tot }}$ and the nuclear slope B as free parameters, to the differential elastic cross section. Taken from Ref. [4].

\section{Inelastic Cross Section at $\sqrt{s}=13 \mathrm{TeV}$}

The measurement of the total inelastic cross section was measured using the first data acquired in Run 2 at $\sqrt{s}=13 \mathrm{TeV}$ (Ref. [12]), with a similar method as detailed in section 3.1. The data were acquired in June 2015 (for a total integrated luminosity of $63 \pm 6 \mathrm{nb}^{-1}$ ) using a trigger provided by the MBTS. It was measured in a fiducial region $\xi>10^{-6}$, i.e. $M_{\mathrm{X}}>13 \mathrm{TeV}$ and then extrapolated to the full phase space. Several MC generators were used both for the data correction and comparison to the final result, and for the extrapolation to the full phase space. These include Pythia8 (with different underlying event generators and PDF sets and different Pomeron flux models for the diffractive componenent), and two generators, EPOS LHC (Ref. [13]) and QGSJET-II (Ref. [14]) specifically developed to model the showering of cosmic rays in the atmosphere. EPOS uses a cut Pomeron model for diffraction and differs from Pythia in the modeling of hadronization and in the description of the underlying event, and it was tuned to the existing LHC data at lower energies. QGSJETII instead describes the Pomeron-Pomeron interaction with the Regge Field Theory. The fraction of singlesided events with respect to the minimum bias sample was measured as discussed in section 3.1 and used to constrain the $\mathrm{MC}$ predictions on the fraction $f_{\mathrm{D}}$ of diffractive events, in a model dependent way. Using the Pythia8 generator a tuned value for $f_{\mathrm{D}}$ between $25 \%$

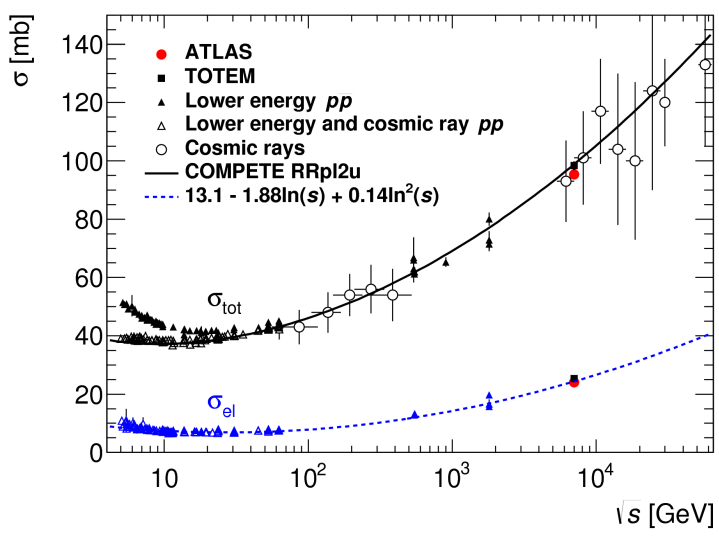

Figure 8: Comparison of total and elastic cross-section measurements with other published measurements and model predictions as function of $\sqrt{s}$. Taken from Ref. [4].

and $32 \%$ is obtained. The result for the fiducial cross section is $\sigma_{\text {inel }}^{\text {fid }}=65.2 \pm 0.8_{\text {exp }} \pm 5.9_{\text {lumi }} \mathrm{mb}$, where the overwhelming contribution to the uncertainty is due to the luminosity determination. The calibration of the luminomters was performed during so-called mini-scans, and not through a full van-der-Meer scan session, and this results in a $9 \%$ calibration uncertainty which will be reduced once the real calibration run, performed one month later, will be fully analysed. The result is between $1 \sigma$ and $1.5 \sigma$ below the predictions of the various generators. In Figure 9 the extrapolation to the full phase space is shown, together with the results at lower energies (from collider data) and at higher energy (from cosmic rays data, Ref. [15]). The extrapolated cross section is measured to be $\sigma_{\text {inel }}=73.1 \pm 0.9_{\exp } \pm$ $6.6_{\text {lumi }} \pm 3.8_{\text {extr }} \mathrm{mb}$ and it is about one standard deviation lower than the theoretical predictions currently available. Additional to the experimental and luminosity uncertainty, the one coming from the extrapolation procedure is added, and it is evaluated by comparing the predictions on the acceptance from the various used MC generators.

\section{Energy Flow}

In order to provide statistically significant samples of hard-scattering processes, which are very rare compared to the dominant low-transfer momentum interactions, LHC normally provides high instantaneous luminosities, resulting in multiple pp interactions to happen simultaneously. Additional to this class of events, due to superimposed multiple pp interactions, another source of soft processes normally happen. These are due to the 


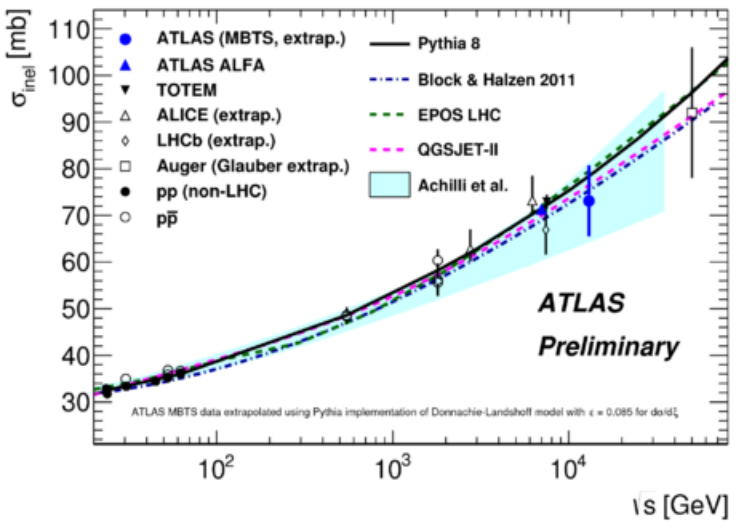

Figure 9: The inelastic cross section versus $\sqrt{s}$. The ATLAS measurements are shown after extrapolation to the full phase space. Taken from Ref. [12].

scattering of the spectator partons, of which the protons are made of, accompanying the main parton-parton interaction producing the hard scattering event. These are normally called underlying events, and are also present, in the context of cosmic ray physics, when a cosmic particle initiates a shower. Both events need to be correctly modelled in the MC generators in order to provide a reliable description of the soft particle kinematics due to the inclusive interactions.

Experimentally ATLAS uses, respectively, a minimum bias trigger to enhance the first class of events (multiple interactions), and a trigger requiring two high$E_{\mathrm{T}}$ jets, to select events where a hard interaction has occurred: by then studying the particle kinematics in the azimuthal region transverse to the hard scatter (leading jet) one is sensitive to the underlying event. The quantity measured (Ref. [16]) is the sum of the transverse energy $\left(\Sigma E_{\mathrm{T}}\right)$ of the particles produced, in bins of the pseudorapidity $\eta$. The transverse energy is measured by the ATLAS calorimeters and is corrected for the detectors effects (at particle level). Both the mean of this quantity (denoted as $<\frac{d^{2} \Sigma E_{\mathrm{T}}}{d \eta d \phi}>$ ) per unit $\eta-\phi$, and its distribution (denoted as $\frac{1}{N_{\text {evt }}} \cdot \frac{d N_{\text {evt }}}{d \Sigma E_{\mathrm{T}}}$ ) are measured up to $|\eta|<4.8$ thanks to the large forward coverage ensured by the calorimeters. The difference between the two used data-sets (minimum-bias and di-jet samples) is that, in the first case $\Sigma E_{\mathrm{T}}$ includes particles at any $\phi$, while in the second sample, only particles transverse with respect to the leading jet $(\pi / 3<|\Delta \phi|<2 \pi / 3)$ are considered in order to enhance the contribution due to the underlying event.

The results are compared to various MC generators, namely Pythia6, Pythia8, Herwig++ and EPOS, all with various tunes. In particular the EPOS LHC version is used, where LHC data are used to tune the parametrization of the evolution of the initial state for the protonproton scattering. As said EPOS is a generator designed to model the development of cosmic showers in air, so that it is particularly important for it to contain all the available knowledge about the accompanying soft interactions.

In Figure 10 the mean value of the $\Sigma E_{\mathrm{T}}$ distribution is shown for the minimum bias trigger sample, after unfolding, as a function of $\eta$. In the forward direction (large $\eta$ ) all MC predictions are too low compared to the data, the better description being given by EPOS. In the case of the di-jet trigger (not shown) also EPOS fails to describe the forward properties. In Figure 11 the $\Sigma E_{\mathrm{T}}$ distribution is shown in the most forward $\eta$ bin, $4.0<\eta<4.8$, using the di-jet trigger. Again all generators fail to fully describe the distribution, in particular for the low and high transverse energy values. In the minimum bias trigger sample (not shown) EPOS is comparable to other generators, while for the di-jet one its discrepancy from the data is evident. Overall all MC predictions are found to understimate the amount of energy in the forward region compared to the central one by as much as 20 to $30 \%$, being EPOS the generator better describing the minimum bias sample.

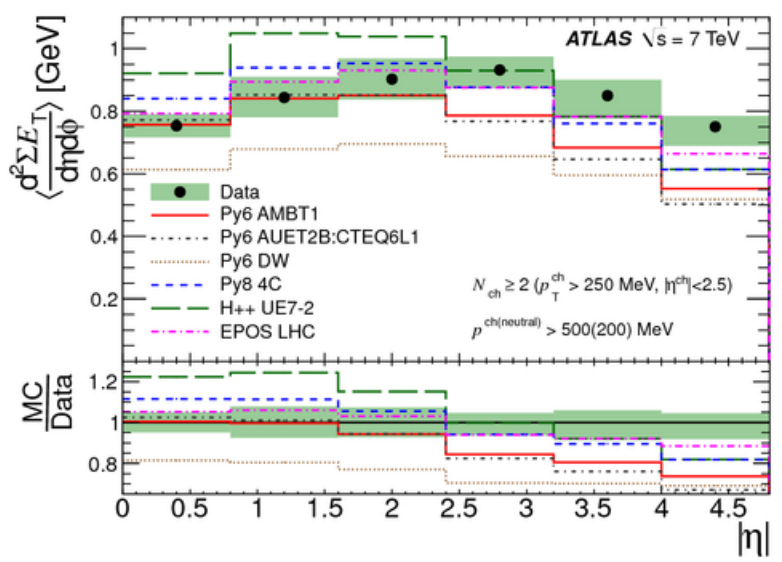

Figure 10: Unfolded $\Sigma E_{\mathrm{T}}$ density distribution compared to various MC models and tunes for the minimum bias selection. Taken from Ref. [16].

\section{Future Perspectives}

During the LHC Run 2 at higher energy $(\sqrt{s}=$ $13 \mathrm{TeV}$ ) ATLAS foresees to perform new measurements of the total, elastic and inelastic cross sections. As from Figure 8 it is clear that such measurement will overlap 


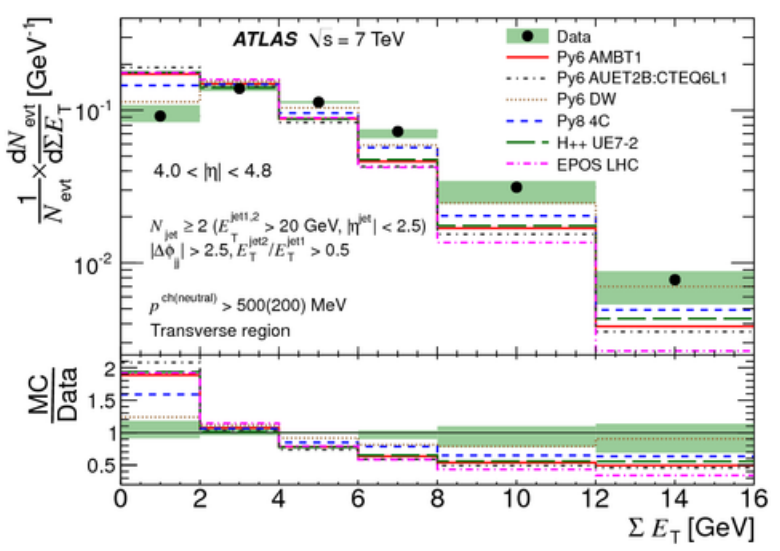

Figure 11: Unfolded $\Sigma E_{\mathrm{T}}$ distribution compared to various MC models and tunes for the dijet selection in the following $4.0<|\eta|<4.8$. Taken from Ref. [16].

with the measurements performed by cosmic rays experiments, but with much better precision. The program will therefore provide a valuable input to cosmic rays physics in a relevant energy range. To perform this program, the ALFA detector has been consolidated in order to cope with values of $\beta^{*}$ as large as $1 \mathrm{Km}$ and be able to probe smaller $|t|$ values and minimize the extrapolation uncertainties.

\subsection{The ATLAS Forward Proton Detector}

One of the major ATLAS upgrades for Run 2 is the Forward Proton Detector, AFP (Ref. [17]), consisting in 2 detector stations in each side of the IP, at about 210 meters from it. Contrary to the ALFA case, the Roman Pots, replica of the TOTEM ones, are horizontal. The detectors will include tracking devices consisting in $3 \mathrm{D}$ silicon pixels deriving from the already working ATLAS Insertable B-layer (IBL). The crucial parameters are the spatial resolution, which was measured to be $10(30) \mu \mathrm{m}$ in the $\mathrm{x}(\mathrm{y})$ directions, a high efficiency, also measured to be larger than $98 \%$, and the radiation hardeness, as the detector will have to survive in a high radiation area. The detectors will be able to approach the beam to about $4 \mathrm{~mm}$ depending on the running optics conditions. Moreover a time-of-flight system is foreseen, with an excellent time resolution, of the order of 10 psec, a large coverage, high efficiency and segmentation, and trigger capabilities. This is of crucial importance to measure, from the arrival time of particles, the position of the proton-proton interaction vertex, and disentangle the various simultaneous interactions happening at high luminosity.

The project was approved in June 2015 by ATLAS and LHC and the schedule foresees the installation of one of the two arms in the 2015-2016 winter shutdown, while the second arm will be installed one year later. During the single arm operation, the time-of-flight detectors are not crucial and will not be installed.

From detailed simulations and based on the experience acquired in the past data takings, AFP seems to be suitable for both the low and the high-luminosity running, both at high and low $\beta^{*}$. This is not the case for ALFA which can only operate at low luminosity. Nevertheless this crucially depends on the background environment, so AFP will initially take data at low luminosity in order to gain experience in this favorable case. In the case of small $\beta^{*}$ LHC optics, AFP shows from simulation a large acceptance in $\xi$ over a large $p_{\mathrm{T}}$ range, contrary to ALFA which spans over a large $\xi$-range down to zero only in the case of large $\beta^{*}$. In this respect ALFA and AFP are therefore complementary in terms of acceptance in the various LHC optics scenarios.

The physics program, in the single-arm configuration, mainly consists in diffractive physics, including soft single-diffraction (cross section measurements and charged particle distributions, of primary interest for the cosmic rays community), and hard single-diffractive jet, $\gamma$ and vector-bosons production. When both arms will be installed a very interesting channel will be accessible, namely the central diffraction, where jets and $\gamma$ are centrally produced via Double-Pomeron exchange between the two diffracted protons.

\section{Conclusions}

ATLAS has performed a wide set of forward physics measurements at $\sqrt{s}=7 \mathrm{TeV}$. Among these, remarkable is the detailed program on cross section measurements (total, elastic and inelastic), differential inelastic cross section, transverse energy distributions in soft interactions, and jet production (not discussed here). The program will be extended in Run 2 at $13 \mathrm{TeV}$, and the first measurements are already available. The results are important for the cosmic rays community, in particular for the modelling of the showers, initiated in air by the cosmic particles of high energy. The measurements at $13 \mathrm{TeV}$ in particular will be fully overlapping with low precision measurements already available from cosmic rays experiments. The ATLAS forward physics program will be futher extended by the new AFP detector, which will allow to perform measurements both at low and high luminosity in the very forward direction.

\section{References}

[1] G. Matthiae, New Journal of Physics, 12 (2010) 075009. 
[2] ATLAS Collaboration, JINST 3, S08003 (2008).

[3] ATLAS Collaboration, Eur. Phys. J. C 73 (2013) 2518, ArXiv:1302.4393.

[4] ATLAS Collaboration, Nuclear Physics B 889 (2014) 486-548, ArXiv: 1408.5778.

[5] ATLAS Collaboration, Nature Commun. 2 (2011) 463, ArXiv:1104.0326.

[6] PYTHIA version 6.421. Sjostrand et al., P.PYTHIA 6.4 Physics and Manual, JHEP 05 (2006) 026-602.

[7] PYTHIA version 8.135. Sjostrand et al., Introduction to Pythia 8.1, Comput. Phys. Commun. 178 (2008) 852-867.

[8] R. Engel and J. Ranft, Phys. Rev. D 54 (1996) 4244-4262.

[9] ATLAS Collaboration, Eur. Phys. J. C72 (2012) 1926, ArXiv: 1201.2808.

[10] G. Antchev et al., Europhys. Lett. 96 (2011) 21002, ArXiv: 1110.1395.

[11] G. Antchev et al., Europhys. Lett. 101 (2013) 21002.

[12] ATLAS collaboration, Measurement of the Inelastic ProtonProton Cross Section at $\sqrt{s}=13 \mathrm{TeV}$ with the ATLAS Detector at the LHC, ATLAS-CONF-2015-038.

[13] T. Pierog et al., EPOS LHC: test of collective hadronization with LHC data (2013) ArXiv: 1306.0121.

[14] S. Ostapchenko, Phys. Rev. D 83 (2011), ArXiv: 1010.1869.

[15] P. Abreu et al., Phys. Rev. Lett. 109 (2012) 062002, ArXiv: 1208.1520.

[16] ATLAS Collaboration, JHEP 11 (2012) 033, ArXiv:1208.6256.

[17] ATLAS Collaboration, ATLAS Forward Proton Phase-I Upgrade. Technical Design Report. CERN-LHCC-2015-009; ATLAS-TDR-024-2015. 